\title{
Optimized Wannier Functions for Hubbard Chain
}

\author{
J. KURZYK ${ }^{a}$, W. WÓJCIK ${ }^{a}$ AND J. SPAŁEK ${ }^{b}$ \\ ${ }^{a}$ Instytut Fizyki Politechniki Krakowskiej \\ Podchorążych 1, 30-084 Kraków, Poland \\ ${ }^{b}$ Instytut Fizyki im. M. Smoluchowskiego, Uniwersytet Jagielloński \\ Reymonta 4, 30-059 Kraków, Poland
}

\begin{abstract}
One-dimensional atomic chain with a variable-range hopping is described within the extended Hubbard model. The Gutzwiller-ansatz approximation is used to determine the optimized single-particle (Wannier) wave functions in the correlated state. Hopping integral up to the third neighbors is taken into account and the results are compared with those for the infinite hopping range. Ground state energy of the system is compared with that making use of the rigorous Lieb-Wu solution with the optimized wave functions. The evolution of the properties as a function of interatomic distance is discussed.
\end{abstract}

PACS numbers: 71.27.+a, 71.30.+h, 71.10.Fd

\section{Introduction}

The infinite one-dimensional atomic chain is described here by the extended Hubbard Hamiltonian

$$
H=\varepsilon_{\mathrm{a}}^{\mathrm{eff}}+\sum_{\langle i j\rangle \sigma} t_{i j} c_{i \sigma}^{\dagger} c_{j \sigma}+U \sum_{i} n_{i \uparrow} n_{i \downarrow},
$$

where $t_{i j}$ - the hopping integral between the sites $i$ and $j, U$ is the magnitude of the Coulomb interaction at given site $i$, and

$$
\varepsilon_{\mathrm{a}}^{\text {eff }} \stackrel{\text { a.u. }}{\equiv} \varepsilon_{\mathrm{a}}+\frac{1}{N} \sum_{i<j}\left(K_{i j}+\frac{2}{R_{i j}}\right)
$$

is the effective atomic energy, $K_{i j}$ is the interaction for electrons located on sites $i$ and $j \neq i$. This energy is important as we study the system evolution as a function of the interatomic distance $R$ [1] and reach the proper atomic limit when $R \rightarrow \infty$. To solve this problem we use the optimized single-particle wave functions method [2]. We construct the Wannier wave functions $\left\{w_{i}(\mathbf{r})\right\}$ entering the expressions for $t_{i j}, U, \varepsilon_{\mathrm{a}}^{\mathrm{eff}}$, and $K_{i j}$ in the following manner:

$$
w_{i}(\boldsymbol{r}) \equiv \sum_{j=1, k=0}^{z_{k}, M} \beta_{k} \Psi_{j}(\boldsymbol{r}),
$$

where $z_{k}$ is the number of atoms of $k$-coordination sphere $(k=1, \ldots, M), \beta_{k}$ are the mixing coefficients, and

$$
\Psi_{i}(\boldsymbol{r}) \equiv \sqrt{\frac{\alpha^{3}}{\pi}} \mathrm{e}^{-\alpha\left|\boldsymbol{r}-\boldsymbol{R}_{i}\right|}
$$

is the $1 s$-like atomic wave function centered around the site $i$. The parameters $\beta_{k}$ are selected to fulfill the orthogonality of the basis $w_{i}(\boldsymbol{r})$. In the previous papers [2] we used the simplest form of the functions (3) with $M=1$. It was sufficient in the tight binding approxima- tion. Now we include the hopping to further atoms so the functions must have more parameters in order to be able to construct the orthogonal basis. We limit to the functions (3) with $M=3$.

We also test the influence of the form of the basis functions for $M=1,2$ and 3 on the ground state energy. For that we make use of the Lieb-Wu solution [3] with ground state energy for site equal to

$$
\frac{E_{\mathrm{G}}}{N}=\varepsilon_{\mathrm{a}}^{\mathrm{eff}}-4 t \int_{0}^{\infty} \frac{J_{0}(\omega) J_{1}(\omega)}{\omega\left(1+\mathrm{e}^{\omega U /(4 t)}\right)} \mathrm{d} \omega,
$$

where $J_{n}(x)$ is the $n$-th order Bessel function.

In concrete calculations we represent the functions (4) by the Gaussians composing the STO-7G basis. In order to achieve the global minimum of the ground state energy the single-particle wave functions are optimized with respect to $\alpha$.

To determine the ground state energy with a variable-range hopping $\left(t_{k} \equiv t_{i, i+k}, \quad k=1,2,3\right)$, we use the Gutzwiller ansatz expression [4]:

$$
\frac{E_{\mathrm{G}}}{N} \equiv \varepsilon_{\mathrm{a}}^{\mathrm{eff}}-|\bar{\varepsilon}|\left(1-U / U_{c}\right)^{2}, \quad U_{\mathrm{c}}=8|\bar{\varepsilon}|,
$$

where $\bar{\varepsilon}$ is the average bare band energy per atom. For small enough hopping integrals $t_{2}$ and $t_{3}$ compared to $t_{1}$, i.e. when a length of the Fermi wave vector is $k_{\mathrm{F}}=\pi / 2$, the average energy $\bar{\varepsilon}$ is equal to

$$
\bar{\varepsilon}=\frac{4}{\pi}\left(t_{1}-t_{3} / 3\right)
$$

Let us note that in this case the average energy $\bar{\varepsilon}$ does not depend on $t_{2}$.

Additionally, we study the case with infinite-range hopping. To do this we consider the model with hopping amplitudes [5]: 


$$
t_{k-l}=\left(-\mathrm{i}\left|t_{1}\right|\right)(-1)^{k-l} \frac{\sinh (\kappa)}{\sinh (\kappa(k-l))},
$$

where $\kappa$ is the effective range of the interaction. In the limit $\kappa \rightarrow \infty$ (and some additional transformation) we obtain the Hubbard model with nearest-neighbor hopping and in the limit $\kappa \rightarrow 0$ and in the thermodynamic limit we obtain the $1 / r$ Hubbard model, with the hopping decaying proportionally to the inverse of the distance. The proper order of limits to take is the thermodynamic limit first, and then $\kappa \rightarrow 0$. In the latter case the dispersion relation

$$
\epsilon(k)=\sum_{r \neq 0} t(r) \mathrm{e}^{-\mathrm{i} k r}
$$

takes the linear form $\epsilon=\pi k t_{1}$ in the whole Brillouin zone.

\section{Results}

The three choices of trial functions (3), with $M=1,2$, and 3 , respectively, show that the functions with $M=2$ and $M=3$ lead to comparable values of the ground state energy (the lowest energy is achieved for $M=3$ ) and are distinctly lower than that for $M=1$. We present the results in Fig. 1. The differences are a consequence of difference of shape of the optimal Wannier functions. The optimal Wannier functions with $M=2$ and $M=3$ are very similar and they differ from the one with $M=1$. The difference is visible mainly in tails of the functions. The three optimized Wannier functions are shown in Fig. 2.

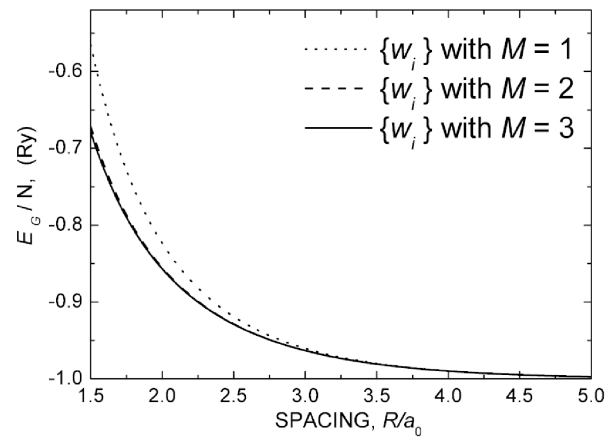

Fig. 1. The ground state energy of the Hubbard chain within the Lieb-Wu solution as a function of interatomic distance for the three forms of starting Wannier functions (with $M=1,2,3$ ).

The ground state energy of the Hubbard chain in the Gutzwiller-ansatz approximation and with the variable-range hopping is shown in Fig. 3. In the case of hopping to nearest neighbors only (tight binding approximation) we show two results. The first is obtained for the simplest trial basis of the Wannier functions $w_{i}(r)$ with $M=1$ and the second for more complicated one for $M=3$. As in the case of the Lieb-Wu solution, the basis with $M=3$ leads to lower energy. When the basis with $M=3$ is used, the ground state energy is the same both in tight binding approximation and in the model with the

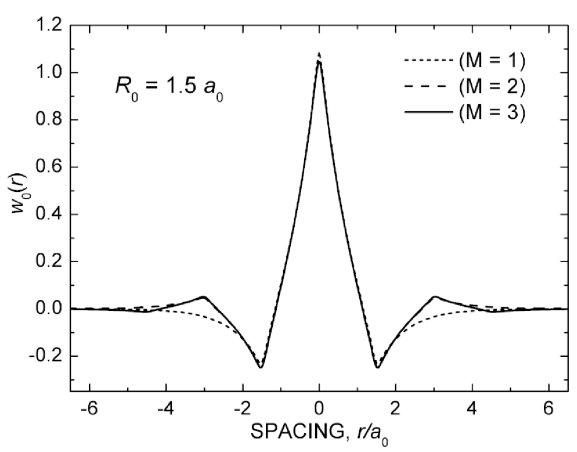

Fig. 2. Exemplary forms of the optimized Wannier functions with $M=1,2$, and 3 for the Lieb-Wu solution. The lattice parameters $R=1.5 a_{0}$ and the parameters of the Wannier functions are: $\alpha=1.332 / a_{0}$ $\beta_{0}=1.435, \beta_{1}=0.472(M=1) ; \alpha=1.290 / a_{0}$, $\beta_{0}=1.507, \beta_{1}=0.550, \beta_{2}=0.111(M=2) ; \alpha=$ $1.281 / a_{0}, \beta_{0}=1.520, \beta_{1}=0.561, \beta_{2}=0.124, \beta_{3}=$ $-0.02741(M=3)$. For details see main text.

hopping up to second neighbors. This results from applying the Gutzwiller ansatz because for all interatomic distances under consideration, the average bare band energy does not depend on $t_{2}$ (see Eq. (7)). The ground state energy for the case of hopping up to third neighbors is higher than for that for lower hopping range. This is also a consequence of Eq. (7) because both $t_{1}$ and $t_{3}$ are negative (cf. Fig. 4). Let us note also the presence of shallow minimum (as marked) in the case with the infinite hopping range.

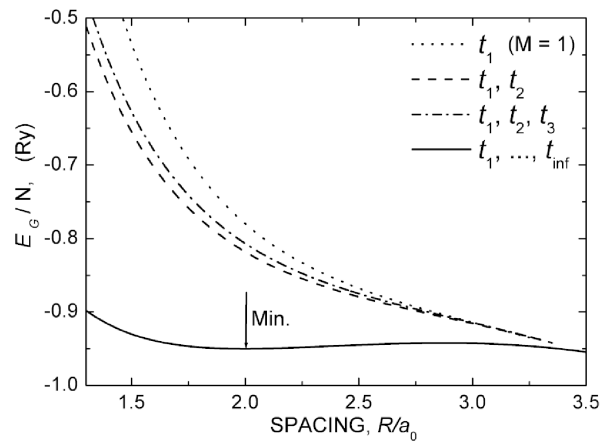

Fig. 3. Ground state energy of the Hubbard chain for the case with variable-range of hopping. Calculations were performed with the $M=3$ basis. For variable hopping the Gutzwiller ansatz was used. For comparison the result for the $M=1$ basis is shown.

The first three hopping integrals for the Hubbard chain as a function of $R$ are plotted in Fig. 4. The first and the third of them are negative, and the second is positive. Absolute values of the ratios of the consecutive hopping integrals are comparable in magnitude, especially for larger interatomic distances. For example, for $R=2.0 a_{0}, t_{2} /\left|t_{1}\right| \approx 0.26$ and $\left|t_{3}\right| / t_{2} \approx 0.24$. 


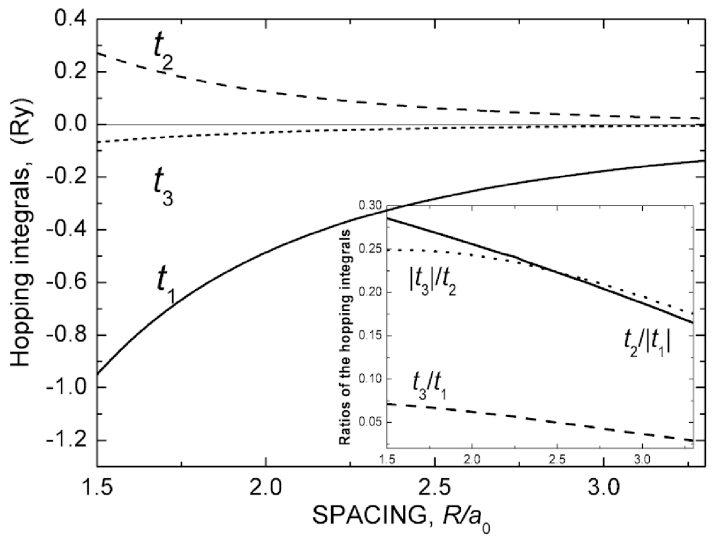

Fig. 4. First three hopping integrals vs. $R$. Inset: ratios of the hopping integrals.

\section{Outlook}

We have determined single-particle (Wannier) wave functions in the correlated state of electrons for the extended Hubbard model with variable-hopping range. Both the exact Lieb-Wu and the Gutzwiller-ansatz solutions have been utilized in appropriate situations (the former is limited only to the case with nearest neighbor hopping). The extension of the atomic basis composing Wannier functions (from $M=1$ fo $M=3$ ) alters the shape of the optimized Wannier functions (cf. Fig. 2), since upon increasing $M$, the orthogonality of more distanct functions can be achieved. The results for $M=2$ and $M=3$ are already convergent.

\section{Acknowledgments}

The work was supported in part by grant No. 1P03B 00129 from Ministry of Science and Higher Education and in part by the Marie Curie TOK grant MTDK-CT-2004-517186 "Correlations in Complex Systems" (COCOS).

\section{References}

[1] J. Kurzyk, J. Spałek, W. Wójcik, Acta Phys. Pol. A 111, 603 (2007).

[2] For review see: J. Spałek, E.M. Görlich, A. Rycerz, R. Zahorbeński, J. Phys., Condens. Matter 19, 255212 (2007); J. Spałek, R. Podsiadły, W. Wójcik, A. Rycerz, Phys. Rev. B 61, 15676 (2000).

[3] E.H. Lieb, F.Y. Wu, Phys. Rev. Lett. 20, 1445 (1968); Physica A 321, 1 (2003); arXiv:cond-mat/0207529, 2002.

[4] M.C. Gutzwiller, Phys. Rev. 137, A1726 (1965); W.F. Brinkman, T.M. Rice, Phys. Rev. B 2, 4302 (1970).

[5] See: F. Gebhard, The Mott Metal-Insulator Transition, Springer, Berlin 1997, p. 168ff. 